\title{
Does Increasing Packing Density Using Larger Caliber Coils Improve Angiographic Results of Embolization of Intracranial Aneurysms at 1 Year: A Randomized Trial
}

\author{
(D). Raymond, (D). Ghostine, (DB.A. van Adel, (D).J.S. Shankar, (DD. lancu, (D) A.P. Mitha, (D) P. Kvamme, (D) R.D. Turner, (D) A. Turk, \\ (D) V. Mendes-Pereira, (D).S. Carpenter, (D). Boo, (D)A. Evans, DH.H. Woo, (DD. Fiorella, (D) A. Alaraj, (DD. Roy, (D)A. Weill, \\ (D) P. Lavoie, (D) M. Chagnon, (D)T.N. Nguyen, (D).L. Rempel, and (D)T.E. Darsaut
}

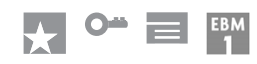

\begin{abstract}
BACKGROUND AND PURPOSE: The impact of increased aneurysm packing density on angiographic outcomes has not been studied in a randomized trial. We sought to determine the potential for larger caliber coils to achieve higher packing densities and to improve the angiographic results of embolization of intracranial aneurysms at 1 year.
\end{abstract}

MATERIALS AND METHODS: Does Embolization with Larger Coils Lead to Better Treatment of Aneurysms (DELTA) was an investigator-initiated multicenter prospective, parallel, randomized, controlled clinical trial. Patients had 4- to 12-mm unruptured aneurysms. Treatment allocation to either 15- (experimental) or 10-caliber coils (control group) was randomized 1:1 using a Web-based platform. The primary efficacy outcome was a major recurrence or a residual aneurysm at follow-up angiography at $12 \pm 2$ months adjudicated by an independent core lab blinded to the treatment allocation. Secondary outcomes included indices of treatment success and standard safety outcomes. Recruitment of 564 patients was judged necessary to show a decrease in poor outcomes from $33 \%$ to $20 \%$ with 15 -caliber coils.

RESULTS: Funding was interrupted and the trial was stopped after 210 patients were recruited between November 2013 and June 2017. On an intent-to-treat analysis, the primary outcome was reached in 37 patients allocated to 15 -caliber coils and 36 patients allocated to 10 -caliber coils (OR $=0.931 ; 95 \% \mathrm{Cl}, 0.528-1.644 ; P=.885)$. Safety and other clinical outcomes were similar. The 15-caliber coil group had a higher mean packing density $(37.0 \%$ versus $26.9 \%, P=.0001)$. Packing density had no effect on the primary outcome when adjusted for initial angiographic results ( $\mathrm{OR}=1.001 ; 95 \% \mathrm{Cl}, 0.981-1.022 ; P=.879)$.

CONCLUSIONS: Coiling of aneurysms randomized to 15-caliber coils achieved higher packing densities compared with 10-caliber coils, but this had no impact on the angiographic outcomes at 1 year, which were primarily driven by aneurysm size and initial angiographic results.

$\mathbf{E}^{\mathrm{n}}$ ndovascular embolization of intracranial aneurysms with platCinum coils has been shown to be effective for preventing rebleeding in aneurysmal SAH and is a widely offered treatment

\section{Received October 16, 2019; accepted after revision November 6.}

From the Department of Radiology (J.R., J.G., D.R., A.W.), Service of Interventional Neuroradiology, Centre Hospitalier de l'Université de Montréal, Montreal, Quebec, Canada; Department of Surgery/Medicine (B.A.v.A), McMaster University, Hamilton, Ontario, Canada; Division of Neuroradiology (J.J.S.S.), Department of Radiology, Dalhousie University, Halifax, Nova Scotia, Canada; Department of Radiology (J.J.S.S.), University of Manitoba, Winnipeg, Manitoba, Canada; Department of Radiology, Service of Interventional Neuroradiology (D.I.), University of Ottawa Hospitals, Civic Campus, Ottawa, Ontario, Canada; Department of Clinical Neurosciences (A.P.M.), University of Calgary, Calgary, Alberta, Canada; Department of Radiology (P.K.), University of Tennessee Medical Center, Knoxville, Tennessee; Department of Neurosurgery (R.D.T., A.T.), Prisma Health-Upstate, Greenville, South Carolina; Division of Neuroradiology (V.M.-P.), Department of Medical Imaging, Toronto Western Hospital, University Health Network, Toronto, Ontario, Canada; Department of Neuroradiology (J.S.C., S.B.), West Virginia University, Rockefeller Neuroscience Institute, Morgantown, West Virginia; Department of Interventional Neuroradiology (A.E.), University of Virginia Medical Center, Charlottesville, Virginia; Departments of Neurosurgery and Radiology, Northwell Health System (H.H.W., D.F.), Manhasset, New York; Department of Neurosurgery (A.A.), University of Illinois Hospital and Health Sciences System, Chicago, Illinois; Department of Neurosurgery (P.L.), Hôpital Enfant-Jésus, Quebec City, Quebec, Canada; Department of Mathematics and Statistics (M.C.), University of Montreal, Montreal, Quebec, Canada; Departments of Neurology, Neurosurgery, and Radiology (T.N.N.), Boston Medical Center, Boston, Massachusetts; and Department of Radiology and Diagnostic Imaging (J.L.R.) and Division of Neurosurgery (T.E.D.), Department of Surgery, Mackenzie Health Sciences Centre, University of Alberta Hospital, Edmonton, Alberta, Canada. for the primary prevention of SAH from unruptured aneurysms. ${ }^{1,2}$ However, aneurysm recurrence following initial treatment is not infrequent, leading to long-term angiographic surveillance and retreatment when aneurysms recur. ${ }^{3}$ A multicenter registry has reported up to $15 \%$ retreatment rates at 2 years after coiling of ruptured aneurysms, but a yearly rerupture rate of only $0.2 \%$ after the first year. ${ }^{4}$ Recurrence has been linked to several factors, including rupture status, aneurysm size, neck width, initial angiographic results of treatment, and packing density. ${ }^{5,6}$ Large-caliber platinum coiling systems (15-caliber coils) have been introduced for use in relatively small aneurysms. The aim was to more completely fill the aneurysm sac with embolic material. ${ }^{7}$ We sought to determine the potential for those larger caliber

Unrestricted research grant from DePuy Synthes, part of Johnson \& Johnson Medical Products, a division of Johnson \& Johnson Inc; and Cerenovus, Part of DePuy Synthes Products Inc (study No. CSS-CNV-14-006).

Please address correspondence to Jean Raymond, MD, Department of Radiology, Service of Interventional Neuroradiology, Centre Hospitalier de l'Université de Montréal, 1000 Saint-Denis St, Room D03-5462B, Montreal, QC H2X 0Cl Quebec, Canada; e-mail: jean.raymond@Umontreal.ca

-- Indicates open access to non-subscribers at www.ajnr.org

$\equiv$ Indicates article with supplemental on-line tables.

http://dx.doi.org/10.3174/ajnr.A6362 
coils to achieve higher packing densities in 4- to 12-mm aneurysms and ultimately to decrease the rate of poor angiographic outcomes at 1 year. The Does Embolization with Larger Coils Lead to Better Treatment of Aneurysms (DELTA) trial was initiated to examine the safety and efficacy of endovascular coil embolization with 15-caliber coils (DELTAMAXX Microcoil; DePuy Synthes, Codman for Neuro, Raynham, Massachusetts) compared with embolization with 10-caliber coils in patients with small-to-medium-sized aneurysms. The prespecified pilot phase reporting the capacity for 15 -caliber coils to increase packing density compared with 10 -caliber coils has been previously published. ${ }^{8}$ The hypothesis of the pivotal study was a decrease in the rate of residual aneurysm and recurrence at 1 year from $33 \%$ with 10 -caliber to $20 \%$ with 15 -caliber coils, necessitating the recruitment of 564 patients ( $\alpha, 5 \% ; \beta$ error, 10\%). Recruitment was stopped on December 5, 2108, because financial support was interrupted despite the recommendation of the trial Data Safety and Monitoring Committee (DSMC) to pursue the trial. We report here the final results of the DELTA trial.

\section{MATERIALS AND METHODS Trial Design}

DELTA was an investigator-initiated multicenter prospective, parallel, randomized, controlled, single-blind clinical trial. The study is registered and summarized on https://clinicaltrials.gov/, NCT01943591, and the full protocol can be found at http://bit.ly/ 2DZcTot. There were no changes to the protocol during the conduct of the trial.

\section{Eligibility Criteria}

The trial had wide inclusion criteria: adult patients with at least 2 years of life expectancy, having a ruptured (World Federation of Neurosurgical Societies grades I-III SAH) or unruptured 4- to 12-mm intracranial aneurysm, considered for endovascular coil embolization and amenable for treatment with either 15- or 10caliber coils. There were few exclusion criteria: patients with planned treatment of an associated cerebral arteriovenous malformation and those for whom parent vessel occlusion was the primary intent of the procedure.

\section{Participating Centers}

The trial was to be conducted in approximately 20 neurovascular centers with experience in endovascular treatment of aneurysms (at least 100 patients previously treated).

\section{Interventions}

Patients allocated to the 15-caliber coiling group were preferably to receive as many 15-caliber platinum coils as safely achievable, but the procedure could be completed with 10-caliber filling or finishing coils, to optimize complete occlusion. Patients allocated to the 10-caliber coiling group were to receive only platinum coils of the latter size. Operators had the technical freedom to use any adjunctive technique, such as balloon-assisted or stent-assisted coil embolization. The study did not require any additional testing or procedure other than routine practice at the participating center.
To explore some explanatory analyses in case of nonconclusive intent-to-treat analyses, we prespecified a coiling protocol deviation to occur in the 15 -caliber coil group when $<30 \%$ of coil length was 15-caliber coils; in the 10-caliber coil group, any use of 15-caliber coils was considered a coiling protocol deviation. Coiling protocol deviations were included in their allocated coil group in intent-to-treat analyses but were removed from their allocated coil group (ie, not considered as crossovers) in the perprotocol analyses.

\section{Randomization}

Randomized (1:1) allocation of treatment groups was concealed using a Web-based platform, and the rupture status and previous treatment of the aneurysm were used as minimization criteria.

\section{Outcomes}

The primary efficacy outcome measure was a composite end point consisting mostly of the occurrence of a major recurrence or a residual aneurysm at the time of follow-up angiography at $12 \pm 2$ months, as adjudicated by an independent core lab blinded to the treatment allocation. The degree of aneurysm occlusion was adjudicated according to the Montreal scale (complete occlusion, residual neck, or residual aneurysm). ${ }^{5}$ In the absence of follow-up imaging, other components of the composite primary end point were adjudicated by an independent committee according to the following prespecified criteria of treatment failures (expected to be infrequent): initial treatment failure, when no coils were deployed; hemorrhage during the follow-up period; retreatment of the same lesion by endovascular or surgical means during the follow-up period; occurrence or progression of mass effect in relation to the treated aneurysm; or treatmentrelated morbidity and mortality precluding follow-up angiography.

Secondary outcomes included indices of immediate treatment success, individual components of the composite primary outcome, and standard safety outcomes: initial technical success of the coiling strategy; use of adjunctive devices; number and total length of coils implanted for each type; packing density; time of fluoroscopic exposure (the addition of the time, in minutes, for both detectors of the biplane angiography unit); immediate angiographic results according to the Montreal scale; major recurrence on follow-up angiography; procedure-related serious adverse events; any adverse event; length of hospital stay; discharge destination; mRS at discharge and at 1-year follow-up; and morbidity and mortality that precluded angiographic follow-up.

\section{Packing Density}

Packing density or volume embolization ratio is the volume of inserted coils divided by the aneurysm volume. Coil volume was calculated using the following formula: $\mathrm{V}=\pi(\mathrm{c} / 2)^{2} \mathrm{~L}$, where $\mathrm{c}$ is coil caliber and $\mathrm{L}$ is coil length for coils entered in the procedural case report forms. Aneurysm volumes were determined using a simple mathematic model: $\mathrm{V}=4 / 3 \pi \mathrm{ab}(\mathrm{a}+\mathrm{b}) / 2$, where $\mathrm{a}$ and $\mathrm{b}$ are half of the long and short axes of the aneurysm, as entered on the procedural case report forms.

\section{Analyses}

Descriptive statistics were obtained on demographic variables and aneurysm characteristics to compare the 2 groups at baseline. 
Means, SDs, and range are presented for quantitative variables, and frequency tables, for categoric variables. Comparability of the groups was assessed through an independent Student $t$ test (quantitative data) or exact $\chi^{2}$ tests (categoric data). The primary outcome, recurrence rates (for both intent-to-treat and per-protocol groups), was compared between groups through $\chi^{2}$ tests and odds ratios with $95 \%$ confidence intervals. Logistic regression was used to examine an association between packing density and primary outcome. Secondary outcomes and safety data were compared between groups through independent Student $t$ tests (quantitative variables) or exact $\chi^{2}$ statistics (categoric data). Finally, logistic regression was used to find variables capable of predicting recurrences. The variables were selected using the stepwise forward approach based on likelihood using $\alpha<.05$ for inclusion. Possible predictors included the type of aneurysm, size of the aneurysm, size of the neck, immediate angiographic results, and packing density. Analyses were performed using SPSS, Version 25 (IBM, Armonk, New York) and a significance level of 5\%.

\section{Trial Conduct}

The trial was sponsored by the Center Hospitalier de l'Université de Montréal and funded by Johnson \& Johnson Medical Products, a division of Johnson \& Johnson and DePuy Synthes. The sponsor and funder had no role in study design, data collection, analysis, or reporting and had no direct or indirect access to the data or source documents. The Steering Committee bears the sole responsibility for all aspects of the trial.

\section{RESULTS}

Between November 2013 and June 2017, two hundred ten patients were recruited in 12 North American centers: One hundred four patients were allocated to the 15-caliber group versus 106 to the 10-caliber group. The trial profile is shown in Fig 1. The baseline patient and aneurysm characteristics of the 2 groups were similar (Table 1).

The primary outcome could be adjudicated in 201 patients (96\%). Results are summarized in Table 2. In the intent-to-treat analysis, the primary outcome was reached in 37 patients allocated to 15-caliber coils compared with 36 patients allocated to 10 -caliber coils $(\mathrm{OR}=0.931 ; 95 \% \mathrm{CI}, 0.528-1.644 ; P=.885)$. Including the "not attributed" cases in the "primary outcome met" category or repeating analyses using per-protocol definitions did not change the results.

There was no significant difference in the use of adjunctive devices between the 2 groups. Stents were used in 19 of 101 or $19 \%$ of patients in the 10-caliber group compared with 23 of 102 or $23 \%$ of patients in the 15 -caliber group $(P=.458)$. For patients treated with stent-assisted coiling, the primary outcome was met in 4 of 19 patients in the 10-caliber group compared with 7 of 23 patients in the 15-caliber group $(P=.726)$.

Safety and other clinical outcomes were similar for the 2 groups (On-line Table 1). Fluoroscopy times were similar (71 \pm 45 versus $64 \pm 38$ minutes, $P=.232$ ). Serious adverse events (Table 3) occurred in 25 patients, 7 in the 15-caliber group and 18 in the 10-caliber group, including 9 deaths, 2 in the 15-caliber and 7 in the 10 -caliber group $(P=.053)$. Other secondary outcomes were similar, except for the mean number of coils, which

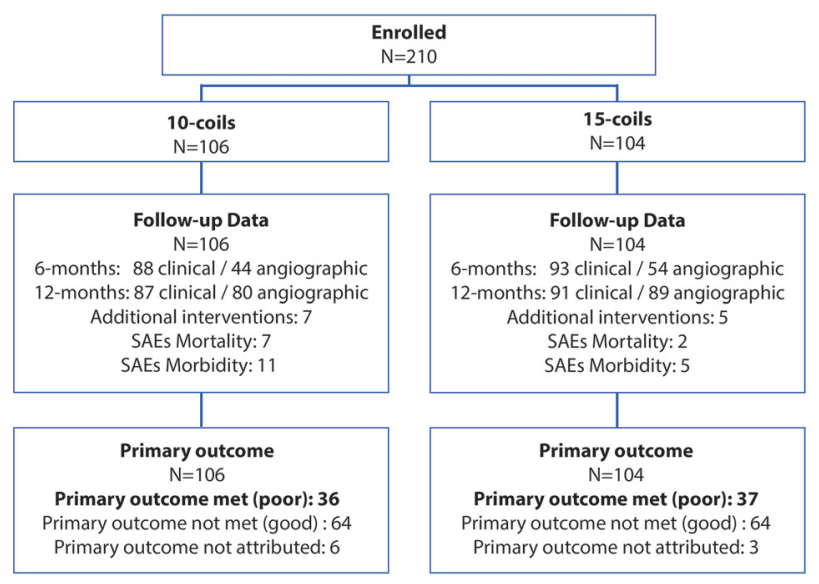

FIG 1. Trial profile. SAEs indicates severe adverse events.

Table 1: Patient demographics and aneurysm characteristics ${ }^{\mathrm{a}}$

\begin{tabular}{|c|c|c|}
\hline & $\begin{array}{c}\text { 10-Caliber } \\
\text { Coils } \\
(n=106)\end{array}$ & $\begin{array}{c}\text { 15-Caliber } \\
\text { Coils } \\
(n=104)\end{array}$ \\
\hline Female sex & $81(76.4 \%)$ & $82(78.8 \%)$ \\
\hline Age (mean) (SD) (yr) & 57 (11) & $58(10)$ \\
\hline mRS at baseline $>2$ & $2(1.9 \%)$ & $3(2.9 \%)$ \\
\hline Multiple aneurysms (No.) (\%) & $29(27.4 \%)$ & $29(27.9 \%)$ \\
\hline \multicolumn{3}{|l|}{ Ruptured aneurysms } \\
\hline $\begin{array}{l}\text { (No.) (\%) of treatment group } \\
\text { (No.) (\%) recurrence }\end{array}$ & $\begin{array}{c}35(33.0 \%) \\
0\end{array}$ & $\begin{array}{c}36(34.6 \%) \\
1(1.0 \%)\end{array}$ \\
\hline \multicolumn{3}{|l|}{ Unruptured aneurysms } \\
\hline (No.) (\%) of treatment group & $71(67.0 \%)$ & $68(65.4 \%)$ \\
\hline (No.) (\%) recurrence & $4(3.8 \%)$ & $3(2.9 \%)$ \\
\hline (No.) (\%) symptomatic & $7(6.6 \%)$ & $5(4.8 \%)$ \\
\hline \multicolumn{3}{|l|}{ Aneurysm size (maximal dimension) } \\
\hline Mean (SD) (mm) & $7.7(2.6)$ & $7.8(2.3)$ \\
\hline \multicolumn{3}{|l|}{ Aneurysm neck } \\
\hline Mean (SD) (mm) & $3.2(1.1)$ & $3.3(1.3)$ \\
\hline Neck $\geq 4.0(\mathrm{~mm})$ & $39(36.8 \%)$ & $31(29.8 \%)$ \\
\hline Aneurysm location, anterior (No.) (\%) & $81(76.4 \%)$ & $88(84.6 \%)$ \\
\hline Internal carotid & $36(34.0 \%)$ & $42(40.4 \%)$ \\
\hline Anterior cerebral & $32(30.2 \%)$ & $31(29.8 \%)$ \\
\hline Middle cerebral & $13(12.3 \%)$ & $15(14.4 \%)$ \\
\hline Aneurysm location, posterior (No.) (\%) & $25(23.6 \%)$ & $16(15.4 \%)$ \\
\hline Basilar & $18(17.0 \%)$ & $12(11.5 \%)$ \\
\hline Other posterior & $7(6.6 \%)$ & $4(3.8 \%)$ \\
\hline
\end{tabular}

${ }^{a}$ No significant difference was found between groups on any of the parameters.

Table 2: Primary outcome $\mathrm{e}^{\mathrm{a}}$

\begin{tabular}{lcc}
\hline & $\begin{array}{c}\text { 10-Caliber Coils } \\
(\boldsymbol{n}=106)\end{array}$ & $\begin{array}{c}\text { 15-Caliber Coils } \\
(\boldsymbol{n}=104)\end{array}$ \\
\hline Primary outcome met & $36(34.0 \%)$ & $37(35.6 \%)$ \\
Major recurrence & $20(18.9 \%)$ & $26(25.0 \%)$ \\
Retreatment & $4(3.8 \%)$ & $4(3.8 \%)$ \\
Initial treatment failure & $6(5.7 \%)$ & $4(3.8 \%)$ \\
SAH & $1(0.9 \%)$ & 0 \\
Related mortality & $5(4.7 \%)$ & $2(1.9 \%)$ \\
Related morbidity & 0 & $1(1.0 \%)$ \\
Good outcome & $64(60.4 \%)$ & $64(61.5 \%)$ \\
Not attributed & $6(5.7 \%)$ & $3(2.9 \%)$ \\
Lost to follow-up & $4(3.8 \%)$ & $3(2.9 \%)$ \\
Unrelated mortality & $2(1.9 \%)$ & 0 \\
\hline
\end{tabular}

${ }^{a}$ No significant difference between groups was found in the primary outcome. Data are (No.) (\%). 
was significantly smaller for 15-caliber coils (On-line Table 2). The 15-caliber coil group had a higher mean packing density (37.0\% versus $26.9 \%, P=.001,2$-tailed $t$ test; excluding cases in which coils could not be deployed). Exploratory per-protocol analyses excluded all coiling protocol violations $(n=21 ; 4$ in the 10 -caliber group, 17 in the 15 -caliber group), initial treatment

Table 3: Serious adverse events

\begin{tabular}{|c|c|c|}
\hline & $\begin{array}{l}\text { 10-Caliber Coils } \\
\quad(n=106)\end{array}$ & $\begin{array}{c}\text { 15-Caliber Coils } \\
(n=104)\end{array}$ \\
\hline Mortality, total (No.) (\%) & $7(6.6 \%)$ & $2(1.9 \%)$ \\
\hline $\begin{array}{l}\text { Aneurysm rupture during } \\
\text { procedure }\end{array}$ & $2(1.9 \%)$ & $1(1.0 \%)$ \\
\hline Stroke, periprocedural & $2(1.9 \%)$ & 0 \\
\hline $\begin{array}{l}\text { Stroke, related to SAH at } \\
\text { presentation }\end{array}$ & $1(0.9 \%)$ & 0 \\
\hline $\begin{array}{l}\text { Related to SAH during } \\
\text { follow-up }\end{array}$ & 0 & $1(1.0 \%)$ \\
\hline $\begin{array}{l}\text { Unrelated to aneurysm or } \\
\text { its treatment }\end{array}$ & $2(1.9 \%)$ & 0 \\
\hline Morbidity, total (No.) (\%) & $11(10.4 \%)$ & $5(4.8 \%)$ \\
\hline $\begin{array}{l}\text { Aneurysm rupture during } \\
\text { procedure }\end{array}$ & $4(3.8 \%)$ & $3(2.9 \%)$ \\
\hline Stroke & $6(5.7 \%)$ & $2(1.9 \%)$ \\
\hline SAH during follow-up & $1(0.9 \%)$ & 0 \\
\hline
\end{tabular}

failures $(n=11)$, and patients with no 12-month follow-up $(n=17)$. In those exploratory analyses, the most important predictor of long-term results was the immediate angiographic result. Other predictors were aneurysm size and rupture status of the aneurysm. Packing density was a significant predictor in univariate analysis, but packing density had no effect on the primary outcome when adjusted for initial angiographic results $(\mathrm{OR}=$ 1.001; 95\% CI, 0.981-1.022; $P=.897)$.

\section{DISCUSSION}

Packing density has been conceived as an estimate of the extent to which an aneurysm is filled with embolic coiling material. ${ }^{9-11}$ It has been thought to play a role in objectively estimating the success of the initial endovascular treatment, at least compared with other, more subjective end points such as angiographic results. ${ }^{12}$ Inferior packing density has been associated with an increased risk of angiographic recurrences in some observational follow-up angiographic studies, ${ }^{13-15}$ but randomized evidence had not been available before the DELTA study. Even though packing density can be calculated with relative ease, its value in predicting inferior follow-up angiographic results remains controversial. ${ }^{16,17}$ Packing density, though objective because based on mathematical calculations, can be predictive of long-term angiographic outcome only after treatment. Packing density is not independent of other risk factors for recurrences that can be determined before treatment, such as aneurysm size, neck width, and the ruptured or unruptured nature of the aneurysm. ${ }^{5,6,14,15,18}$ In addition, if there is an association between packing density and immediate angiographic results, there are intuitive and empiric reasons to believe that immediate angiographic results are causally more pertinent to treatment success, in the long term, than packing density (Fig 2).

It would not be possible to undertreat or overtreat aneurysms in a deliberate, prospective, and randomized fashion, using the same platinum coil technology, keeping all other variables similar, to experimentally study the effects of packing density on longterm results. However, comparing 2 groups of patients with relatively small aneurysms randomly allocated to the exclusive use of 10-caliber platinum coils, considered the control group, or to as many 15-caliber coils as possible, considered the experimental group, keeping all other treatment variables the same, allowed us to examine the potential effects of packing density isolated from all other causal factors.
FIG 3. Effect of packing density and immediate angiographic results. Packing density is better thought of as an index compounding many causal factors, such as aneurysm and neck sizes and immediate angiographic results, rather than a true causal factor in long-term angiographic results. 
Packing density is not an end in itself, but it was used as an end point of the pilot phase because it was thought that if the use of larger caliber coils (the only difference between the 2 platinum coil treatment groups) could not impact packing density, it would be unlikely to change the treatment substantially enough to impact long-term angiographic results.

The use of larger caliber coils did succeed in increasing packing density, ${ }^{8}$ but this had no impact on the primary outcome of the trial, the 1-year angiographic results. We must, of course, remember that the trial was interrupted prematurely. Nevertheless, our exploratory per-protocol data provide experimental evidence supporting an explanation regarding the potentially misleading idea that increasing packing density by itself improves the long-term results of coil embolization (Fig 2). While univariate analyses reveal a significant association between packing density and long-term results, there are also strong associations between aneurysm and neck sizes (predictive factors before treatment) and packing density. In addition, when adjusted for immediate angiographic results, packing density had no influence on results. In other words, packing density is more correctly an indicator rather than a true causal factor in recurrences (Fig 3).

Some authors have argued that packing density is not such an important factor when aneurysms are stented. ${ }^{19,20}$ The subgroup of stented aneurysms in DELTA was too small to draw any conclusions.

Our study has several limitations. The trial was interrupted before the target number of patients had been recruited. The resulting study cannot provide definitive conclusions. The follow-up period was relatively short. The method used to calculate aneurysm volumes is not as accurate as more exact experimental methods, ${ }^{6,21}$ but it is simple, immediately available, and in common clinical usage. Similar methods have been used in most published trials. ${ }^{22}$ This method likely results in an overestimation of aneurysm volumes and an underestimation of packing densities, but it is unlikely to introduce bias in the comparison because of random allocation and because measurement errors are expected to be the same for both groups.

\section{CONCLUSIONS}

Coiling of aneurysms randomized to 15-caliber coils achieved higher packing densities compared with 10-caliber coils, but this finding had no impact on the angiographic outcomes at 1 year, which were primarily driven by aneurysm size and initial angiographic results.

Disclosures: Jimmy Ghostine-RELATED: Grant: Johnson \& Johnson, Comments: unrestricted grant, UNRELATED: Consultancy: Johnson \& Johnson, Comments: short-term consultancy, January 2014, Codman for Neuro, invited speaker at Johnson \& Johnson National Sales Meeting; 1-time honorarium of $2500 \$$ plus travel from Montreal to Toronto and 1-night lodging. Alim P. MithaUNRELATED: Consultancy: Cerus Endovascular, Stock/Stock Options: Fluid Biotech Inc, Comments: cofounder. Miguel Chagnon-UNRELATED: Consulting Fee or Honorarium: University of Montreal.* Raymond Turner-UNRELATED: Consultancy: J\&J/Cerenovus, Siemens, Q'APEL, Rebound Tx, Corindus Vascular Robotics; Patents (planned, pending or issued): Medical University of South Carolina; Royalties: Medical University of South Carolina; Stock/stock options: Corindus Vascular Robotics, Rebound Tx, Endostream, Echovate, Synchron. Thanh Nguyen-David Fiorella-RELATED: Grant: Medtronic, MicroVention, Penumbra, Siemens*; Consulting Fee or Honorarium: Medtronic, MicroVention, Penumbra, Balt; Support for Travel to Meetings for the Study or Other Purposes: Medtronic, MicroVention, Penumbra, Balt; UNRELATED: Board Membership: Vascular Simulation; Consultancy: Medtronic, MicroVention, Penumbra, Siemens, Balt; Expert
Testimony: None; Grants/Grants Pending: Balt, MicroVention, Siemens, Penumbra*; Payment for Lectures Including Service on Speakers Bureaus: Medtronic, MicroVention, Penumbra, Siemens, Stryker, Balt; Patents (Planned, Pending or Issued): Cerenovus, Vascular Simulations; Royalties: Cerenovus; Payment for Development of Educational Presentations: Medtronic; Stock/Stock Options: Neurogami Medical, Marblehead Medical; Travel/Accommodations/Meeting Expenses Unrelated To Activities Listed: MicroVention, Stryker, Balt, Medtronic. Ali Alaraj—UNRELATED: Consultancy: Cerenovus; Grants/Grants Pending: National Institutes of Health.* A. Turk—UNRELATED: Board Membership: Blinktbi; Expert Testimony: Corindus Vascular Robotics, Imperative Care (Corindus Vascular Robotics)*; Patents (Planned, Pending or Issued): Navecap*; Stock/Stock Options; Travel/Accommodations/Meeting Expenses Unrelated to Activities Listed: Hemo China. Sohyun Boo-UNRELATED: Consultancy: Stryker, Microvention, Comments: Education/training. *Money paid to the institution.

\section{ACKNOWLEDGMENT}

The principal acknowledgment must go to the patients, the investigators and the staff who participated in this trial. We also wish to acknowledge the coordination and management team of the study: Guylaine Gevry, Suzanne Nolet, and Ruby Klink.

\section{REFERENCES}

1. Molyneux A, Kerr R, Stratton I, et al. International Subarachnoid Aneurysm Trial (ISAT) of neurosurgical clipping versus endovascular coiling in $\mathbf{2 1 4 3}$ patients with ruptured intracranial aneurysms: a randomized trial. Lancet 2002;360:1267-74 CrossRef Medline

2. Raymond J, Darsaut TE, Molyneux AJ; TEAM Collaborative Group. A trial on unruptured intracranial aneurysms (the TEAM trial): results, lessons from a failure and the necessity for clinical care trials. Trials 2011;12:64 CrossRef Medline

3. Lecler A, Raymond J, Rodriguez-Régent C, et al. Intracranial Aneurysms: Recurrences More than 10 Years after Endovascular Treatment-A Prospective Cohort Study, Systematic Review, and Meta-Analysis. Radiology 2015;277(1):173-80 CrossRef Medline

4. CARAT Investigators. Rates of delayed rebleeding from intracranial aneurysms are low after surgical and endovascular treatment. Stroke 2006;37:1437-42 Medline

5. Raymond J, Guilbert F, Weill A, et al. Long-term angiographic recurrences after selective endovascular treatment of aneurysms with detachable coils. Stroke 2003;34:1398-1403 CrossRef Medline

6. Sluzewski M, van Rooij WJ, Slob MJ, et al. Relation between aneurysm volume, packing, and compaction in 145 cerebral aneurysms treated with coils. Radiology 2004;231:653-58 CrossRef Medline

7. Quasar Grunwald I, Molyneux A, Kuhn AL, et al. Influence of coil geometry on intra-aneurysmal packing density: evaluation of a new primary wind technology. Vasc Endovascular Surg 2010;44:289-93 CrossRef Medline

8. Cloutier F, Khoury N, Ghostine J, et al. Embolization with largercaliber coils can increase packing density: evidence from the pilot phase of a randomized trial. Interv Neuroradiol 2017;23:14-17 CrossRef Medline

9. Reul J, Spetzger U, Weis J, et al. Endovascular occlusion of experimental aneurysms with detachable coils: influence of packing density and perioperative anticoagulation. Neurosurgery 1997;41:116065; discussion 1165-68 Medline

10. Piotin M, Mandai S, Murphy KJ, et al. Dense packing of cerebral aneurysms: an in vitro study with detachable platinum coils. AJNR Am J Neuroradiol 2000;21:757-60 Medline

11. Uchiyama N, Kida S, Nomura $M$, et al. Significance of volume embolization ratio as a predictor of recanalization on endovascular treatment of cerebral aneurysms with Guglielmi detachable coils. Interv Neuroradiol 2000;6(Suppl 1):59-63 CrossRef Medline

12. Tollard E, Darsaut TE, Bing F, et al. Outcomes of endovascular treatments of aneurysms: observer variability and implications for interpreting case series and planning randomized trials. AJNR Am J Neuroradiol 2012;33:626-31 CrossRef Medline

13. Knap D, Gruszczyń ska K, Partyka R, et al. Results of endovascular treatment of aneurysms depending on their size, volume and coil 
packing density. Neurol Neurochir Pol 2013;47:467-75 CrossRef Medline

14. Leng B, Zheng Y, Ren J, et al. Endovascular treatment of intracranial aneurysms with detachable coils: correlation between aneurysm volume, packing, and angiographic recurrence. J Neurointerv Surg 2014;6:595-99 CrossRef Medline

15. Mascitelli JR, Oermann EK, De Leacy RA, et al. Predictors of treatment failure following coil embolization of intracranial aneurysms. J Clin Neurosci 2015;22:1275-81 CrossRef Medline

16. Piotin M, Spelle L, Mounayer C, et al. Intracranial aneurysms: treatment with bare platinum coils-aneurysm packing, complex coils, and angiographic recurrence. Radiology 2007;243:5000-8 CrossRef Medline

17. Goddard JK, Moran CJ, Cross DT, 3rd, et al. Absent relationship between the coil-embolization ratio in small aneurysms treated with a single detachable coil and outcomes. AJNR Am J Neuroradiol 2005;26:1916-20 Medline

18. Sadato A, Adachi K, Hayakawa M, et al. Effects of anatomic characteristics of aneurysms on packing density in endovascular coil embolization: analysis of a single center's experience. Neurosurg Rev 2016;39:109-14; discussion 114 CrossRef Medline

19. Chalouhi N, Dumont AS, Hasan D, et al. Is packing density important in stent-assisted coiling? Neurosurgery 2012;71:381-86 CrossRef Medline

20. Park YK, Bae HJ, Cho DY, et al. Risk factors for recurrence and retreatment after endovascular treatment of intracranial saccular aneurysm larger than $\mathbf{8} \mathbf{~ m m}$. Acta Neurochir (Wien) 2019;161:93946 CrossRef Medline

21. Mascitelli JR, Patel AB, Polykarpou MF, et al. Analysis of early angiographic outcome using unique large diameter coils in comparison with standard coils in the embolization of cerebral aneurysms: a retrospective review. J Neurointerv Surg 2015;7:126-30 CrossRef Medline

22. White PM, Lewis SC, Gholkar A, et al. Hydrogel-coated coils versus bare platinum coils for the endovascular treatment of intracranial aneurysms (HELPS): a randomised controlled trial. Lancet 2011; 377:1655-62 CrossRef Medline 